Results: Pathological changes of the one or two systems were noted in $19.51 \%$ of patients, between three and four systems - in $24,4 \%$, and in some cases - five $(7.32 \%)$ and six systems $(2.44 \%)$.

The mean values of $\mathrm{Cl}$ among patients with SLE were $2.6 \pm 0.3$ points and did not differ significantly depending on the duration of the disease $(2.5 \pm 0.7$ points before 3 years and $2.4 \pm 0.3$ points after 3 years; $p>0.05)$, as well as did not differ in female and male patients $(2.6 \pm 0.3$ and 2.8 \pm 0.5 respectively, $p>0.05$ ). The IC was higher in the cases of development of SLE before the age of 7 years than in older patients $(3.6 \pm 0.5$ vs $2.4 \pm 0.3$ respectively, $\mathrm{p}<0.05)$.

The activity of the pathological process significantly influenced the indicators of the $\mathrm{Cl}$ : $2.4 \pm 0.4$ with its minimal activity; $2.7 \pm 0.4$ with an average degree of activity and $3.0 \pm 0.6$ with a high degree $(p<0.05)$. The frequency of the smallest $\mathrm{Cl}(0$ or 1$)$ turned out to be associated with the lowest activity of the disease.

The $\mathrm{Cl}$ with ANA-positivity association was defined: $2.8 \pm 0.3$ points among patients with ANA that has twice exceeded the $\mathrm{Cl}$ of ANA-negative patients $(1.4 \pm 0.5), p<0,05)$. Its highest level (like 4,5 and 6 points) was registered only among the ANA-positive patients.

In patients with SLE with high $\mathrm{Cl}$, changes in the system of coagulation due to elevated fibrinogen levels predominated $(3.7 \pm 0.4 \mathrm{~g} / \mathrm{l}$ vs $2.8 \pm 0.3 \mathrm{~g} / \mathrm{l}$ $(p<0.05)$. Similar changes were recorded with the atherogenic coefficient increased $(2.7 \pm 0.7$ vs $1.3 \pm 0.5 ; p<0,05)$.

Conclusion: According to the research, it was found that in children and adolescents with SLE the formation of lesions of systems and organs occurs while maintaining the activity of the process, especially immunological (ANA-positivity). Disruption of lipid metabolism and blood coagulation were associated with the formation of comorbid states.

Disclosure of Interests: None declared

DOI: 10.1136/annrheumdis-2019-eular.3808

\section{AB1050 TOCILIZUMAB MODIFIES CLINICAL MANIFESTATIONS AND LABORATORY FEATURES OF SYSTEMIC JUVENILE IDIOPATHIC ARTHRITIS ASSOCIATED MACROPHAGE ACTIVATION SYNDROME}

Masaki Shimizu ${ }^{1}$, Mao Mizuta $^{1}$, Takahiro Yasumi $^{2}$, Naomi Iwata $^{3}$, Yuka Okura $^{4}$, Noriko Kinjo $^{5}$, Hiroaki Umebayashi ${ }^{6}$, Tomohiro Kubota ${ }^{7}$, Yasuo Nakagishi ${ }^{8}$, Kenichi Nishimura ${ }^{9}$, Masato Yashiro ${ }^{10}$, Junko Yasumura ${ }^{11}$, Hiroyuki Wakiguchi ${ }^{12}$, Nami Okamoto ${ }^{13}$, Masaaki Mori ${ }^{14} .{ }^{1}$ Graduate School of Medical Sciences, Kanazawa University, Kanazawa, Japan; ${ }^{2}$ Kyoto University Graduate School of Medicine, Kyoto, Japan; ${ }^{3}$ Aichi Children's Health and Medical Center, Obu, Japan; ${ }^{4}$ KKR Sapporo Medical Center, Sapporo, Japan; ${ }^{5}$ University of the Ryukyus, Nakagami-gun, Japan; ${ }^{6}$ Miyagi Children's Hospital, Sendai, Japan; ${ }^{7}$ Graduate School of Medical and Dental Sciences, Kagoshima University, Kagoshima, Japan; ${ }^{8}$ Hyogo Prefectural Kobe Children's Hospital, Kobe, Japan; ${ }^{9}$ Yokohama City University Graduate School of Medicine, Yokohama, Japan; ${ }^{10}$ Okayama University Hospital, Okayama, Japan; ${ }^{11}$ Hiroshima University Graduate School of Biomedical and Health Sciences, Hiroshima, Japan; ${ }^{12}$ Yamaguchi University Graduate School of Medicine, Ube, Japan; ${ }^{13}$ Osaka Medical College, Takatsuki, Japan; ${ }^{14}$ Graduate School of Medical and Dental Sciences, Tokyo Medical and Dental University, Department of Lifetime Clinical Immunology, Tokyo, Japan

Background: Previous studies including a systematic literature review revealed clinical manifestations and laboratory features of systemic juvenile idiopathic arthritis (s-JIA) associated macrophage activation syndrome (MAS) could be modified in patients treated with tocilizumab (TCZ) ${ }^{1,2}$.

Objectives: To clarify whether TCZ modifies clinical manifestations and laboratory features of S-JIA associated MAS, and to assess performance of the 2016 MAS classification criteria for patients with s-JIA associated MAS while treated with TCZ in the real world.

Methods: A combination of expert consensus and analysis of real patient data was conducted by a panel of 15 pediatric rheumatologists. Clinical manifestations and laboratory features of S-JIA associated MAS at the MAS diagnosis in 12 patients while treated with TCZ and 18 patients not treated with TCZ were evaluated. Possible MAS was defined as having characteristic laboratory features but lack of clinical features of MAS, or atypical MAS, or early treatment that prevented fulminant MAS ${ }^{3,4}$

Results: Among 12 patients while treated with TCZ, only 2 patients were diagnosed with definite MAS, and other 10 patients were diagnosed with possible MAS. On the other hand, among 18 patients not treated with TCZ, 10 patients were diagnosed with definite MAS, and other 8 patients were diagnosed with possible MAS. MAS classification criteria could classify the patients diagnosed with definite MAS while treated with TCZ as having MAS as well as the patients not treated with TCZ $(100 \%$ and $100 \%$, respectively). However, this criteria were less likely to classify the patients diagnosed with possible MAS while treated with TCZ as well as the patients not treated with TCZ $(60 \%$ and $75 \%$, respectively).
Furthermore, the patients with possible MAS while treated with TCZ were less likely febrile and significantly less often had rash, and had notably lower ferritin levels (587 versus $8518 \mathrm{ng} / \mathrm{ml}$; $\mathrm{P}=0.0021$ ), compared to the patients with possible MAS not treated with TCZ. Other laboratory features of MAS including lower platelet counts, lower fibrinogen were more pronounced in patients treated with TCZ.

Conclusion: These findings show TCZ could modify clinical manifestations and laboratory features of s-JIA associated MAS. When evaluating s-JIA patients while treated with TCZ, care must be taken to not underdiagnose MAS based on MAS classification criteria.

\section{REFERENCES}

[1] Shimizu M, et al. Cytokine 2012;58:287-294.

[2] Sculert GS, et al. Arthritis Care Res 2018;70:409-419

[3] Grom AA, et al. Arthritis Rheumatol 2016;68:218-228.

[4] Yokota S, et al. J Rheumatol 2015;42:712-722.

Disclosure of Interests: Masaki Shimizu: None declared, Mao Mizuta: None declared, Takahiro Yasumi Shareholder of: Takeda, Speakers bureau: AbbVie, Novartis, CSL Behring, Naomi Iwata: None declared Yuka Okura: None declared, Noriko Kinjo: None declared, Hiroaki Umebayashi Speakers bureau: AbbVie, Eisai, Novartis, Ono, Chugai, Tomohiro Kubota: None declared, Yasuo Nakagishi: None declared, Kenichi Nishimura: None declared, Masato Yashiro: None declared, Junko Yasumura: None declared, Hiroyuki Wakiguchi: None declared, Nami Okamoto: None declared, Masaaki Mori Grant/research support from: Tokyo Medical and Dental University (TMDU) received unrestricted research grants for Department of Lifetime Clinical Immunology from AbbVie GK, Ayumi Pharmaceutical Corporation, Chugai Pharmaceutical Co., Ltd., CSL Behring K.K. Japan Blood Products Organization, Mitsubishi Tanabe Pharma Corporation, Nippon Kayaku Co., Ltd., Ono Pharmaceutical Co., Ltd., Towa Pharmaceutical Co., Ltd., UCB Japan Co. Ltd.

DOI: 10.1136/annrheumdis-2019-eular.5479

\section{\begin{tabular}{|l|l}
\hline AB1051 TRANSITION CARE IN A NEW PEDIATRIC \\
\hline
\end{tabular} RHEUMATOLOGY CENTER IN BRAZIL: CARING FOR ADOLESCENCE BEYOND RHEUMATISM}

Larissa Pinho, Marco Felipe Silva, Ana Paula Pessoa, Carlos Nobre Rabelo Junior. Hospital Geral de Fortaleza, Pediatric Rheumatology Unit, Fortaleza, Brazil

Background: Transition care is the planned transfer of adolescents with chronic health conditions from children-centered to adult-oriented health systems. Its challenges include gaps in continuity of care, poor adherence, delays in adult services attendance, differences between pediatric and adult centers, difficulties in autonomy over care and unstable medica conditions. EULAR/PRES recently published recommendations, standards and quality indicators on transitional care for young people with rheumatic diseases, which serve as a guide for transitional outpatient clinics. In Brazil, few centers focus on transition from pediatric rheumatology. The Pediatric Rheumatology Unit of Hospital Geral de Fortaleza is a reference in North and Northeast of the country, intending to be pioneer in transitional medicine in region.

Objectives: To evaluate the adolescents and their families' perception regarding follow-up and autonomy in health care, as well as to identify the main risks inherent to this age group.

Methods: A longitudinal and descriptive study was carried out through interviews, questionnaires and medical records analyzes. Adolescents between 15 and 18 years old, regularly followed, who had at least three planned visits/year were included. Patients with cognitive impairment or without definite diagnosis were excluded. Epidemiological and clinical data were collected; self-management and health care utilization skills were evaluated by Transitional Readiness Assessment Questionnaire (TRAQ), a self-administered questionnaire.

Results: Eighty two patients were recruited into the transitional service (61 female [74.4\%]). Patients' ages ranged from 15.0 to 18.7 years (median 16.9). Regarding religion, 41 patients $(54.7 \%)$ were Catholic and 27 were Protestant $(36 \%)$. Concerning to ethnicity, most of them declared themselves brown (61\%) or white (22\%). Fifty-nine adolescents $(72 \%)$ considered themselves able to take care of their own follow-up, whereas 39 relatives $(50 \%)$ considered the patient capable of doing so. The most frequent diseases were juvenile idiopathic arthritis $(31.7 \%)$ and systemic lupus erythematosus (29.3\%), of which $37(45.1 \%)$ were in remission. Median disease duration and follow-up were 48.5 (5 - 180) and 34.5 (1

175) months, respectively. The median TRAQ value was 3.27 (1.61 4.55). There were 191 transitional visits (median 2.0 visits/patient). Fifty three patients had 2 or more visits, out of which $42(79.2 \%)$ had at 
least one risk identified. The most prevalent risks were: overweight or obesity $(31.7 \%)$, sedentarism $(26.4 \%)$, anxiety $(22.6 \%)$, changes in family structure $(22.6 \%)$, school problems $(20.8 \%)$, depression $(18.9 \%)$, suicidal ideation/self-mutilation (18.9\%), low self-esteem (15.1\%). Risks related to psychiatric diseases were identified in 23 patients $(43.4 \%)$.

Conclusion: This is one of the first studies on transition care in Brazil focused on risks identification in adolescence. Transition care allows improvement survival and quality of life due to advances in preventive medicine and treatment of chronic rheumatological diseases. The identification of inherent risks, especially psychiatric disorders, plays a fundamental role in the long-term follow-up, making it possible to deliver multidisciplinary and rehabilitation of adolescents.

\section{REFERENCES}

[1] Ardoin SP. Transitions in Rheumatic Disease: Pediatric to Adult Care. Pediatr Clin North Am. 2018;65(4):867-883.

[2] Foster HE, et al. EULAR/PReS standards and recommendations for the transitional care of young people with juvenile-onset rheumatic diseases. Ann Rheum Dis. 2017;76(4):639-646.

[3] Wood DL, et al. The Transition Readiness Assessment Questionnaire (TRAQ): its factor structure, reliability, and validity. Acad Pediatr. 2014;14 (4):415-22.

Disclosure of Interests: None declared

DOI: 10.1136/annrheumdis-2019-eular.3760

\section{AB1052 SYSTEMIC LUPUS ERYTHEMATOSUS IN CONTEXT OF HUMAN IMMUNODEFICIENCY VIRUS INFECTION: A CLINICAL CONUNDRUM}

Ankita Singh ${ }^{1}$, Anjani Gummadi ${ }^{1}$, Laxmi Makam ${ }^{2}$, Deepti Suri ${ }^{1}$, Anju Gupta ${ }^{1}$, Pandiarajan Vignesh ${ }^{1}$, Ankur Jindal ${ }^{1}$, Amit Rawat ${ }^{1}$, Surjit Singh ${ }^{1} .{ }^{1}$ Post Graduate Institute of Medical Education and Research, Chandigarh, Advanced Pediatrics Centre, Allergy Immunology Unit, Chandigarh, India; ${ }^{2}$ Post Graduate Institute of Medical Education and Research, Chandigarh, Advanced Pediatrics Centre, Chandigarh, India

Background: Systemic lupus erythematosus (SLE) and Human immunodeficiency virus (HIV) infection can occasionally coexist in a patient (1) Also, in rare scenarios, the clinical symptomatology and laboratory tests of these diseases can masquerade each other (2)

Objectives: To describe diagnostic and therapeutic dilemmas of coexistent SLE and HIV

Methods: We analyzed case records of 2 patients with childhood SLE who had the clinical conundrum of HIV infection as well

Results: Case 1- An 11-year-boy presented with large joint arthralgia fever and respiratory distress for 4 days. On examination he had pallor, oral thrush,and onycomycosis. A malar rash was also noted. Systemic examination revealed consolidation in right lung and hepatosplenomegaly. Investigations showed severe anemia and lymphopenia. HIV serology was strongly reactive and CD4 counts were low (49 cells/ $\mu \mathrm{l})$. In view of malar rash he was investigated for SLE and anti-nuclear antibody (ANA) by indirect immunoflorescence (IIF) was 4+ homogenous with rim enhancement. Anti double stranded DNA (dsDNA) antibody titer was $1200 \mathrm{IU} / \mathrm{ml}$, serum complements were low (C3- $<28 \mathrm{mg} / \mathrm{dl}$; C4-10 mg/dl) and direct Coombs test for IgG was positive. Antiphospholipid antibody titers were elevated (Anticardiolipin antibody- Ig G: $695 \mathrm{U} / \mathrm{L}$; anti b2 glycoprotein antibody lg G: 3177 U/L). Blood culture grew Streptococcus pneumoniae. He was started on ceftriaxone and amphotericin, however, he had an acute neurological worsening (raised intracranial pressure) during hospital stay. A diagnosis of concomitant HIV infection and neuropsychiatric SLE was considered in this child. Pulse methylprednisolone was given, but he soon succumbed to the complications.

Case 2: An 8-year-old girl was admitted with fever for 6 months and cough and respiratory distress for 15 days. On examination she had anascarca, oral ulcers, hyperpigmented macules over face and crepitations in right lung fields. Her HIV ELISA was intermediate reactive. Considering a clinical possibility of secondary immunodeficiency due to HIV infection she was managed with broad spectrum antimicrobials while awaiting confirmatory tests for HIV infection. She went on to develop hypertension and nephritis. ANA was 4+ homogenous, low complements and elevated anti dsDNA. Further tests for confirmation of HIV infection showed CD4 counts - 421 cells/ $\mu$, negative HIV viral loads, negative HIV DNA PCR and p24 antigen was negative, so it was considered that the intermediate reactivity of HIV serology was false positive. Subsequently she was given immunosuppression (methylprednisolone pulse and cyclophosphamide) to which she transiently responded however she developed acute intracranial bleed during hospital stay and died.

Conclusion: These cases highlight the fact that serological tests can be difficult to interpret in patients with overlapping manifestations of HIV infection and SLE. However, early diagnosis and prompt therapy is imperative.

\section{REFERENCES}

[1] Burton J, Vera JH, Kapembwa M. HIV and systemic lupus erythematosus: the clinical and diagnostic dilemmas of having dual diagnosis. Int J STD AIDS. 2010 Dec;21(12):845-6.

[2] Carugati M, Franzetti M, Torre A, Giorgi R, Genderini A, Strambio de Castilla $F$, et al. Systemic lupus erythematosus and HIV infection: a whimsical relationship. Reports of two cases and review of the literature. Clin Rheumatol. 2013 Sep;32(9):1399-405

Disclosure of Interests: None declared

DOI: 10.1136/annrheumdis-2019-eular.6739

\section{AB1053 MACROPHAGE ACTIVATION SYNDROME AS A PRESENTATION IN PEDIATRIC LUPUS: A RETROSPECTIVE STUDY OF 3 CASES}

Ankita Singh, Anjani Gummadi, Rakesh Kumar, Sandesh Guleria, Johnson Nameirakpam, Pandiarajan Vignesh, Ankur Jindal, Deepti Suri, Amit Rawat, Surjit Singh. Post Graduate Institute of Medical Education and Research, Chandigarh, Advanced Pediatrics Centre, Allergy Immunology Unit, Chandigarh, India

Background: Macrophage activation syndrome (MAS) can, at times, be the presentation of pediatric lupus and diagnosis requires high index of suspicion.

Objectives: To report children who had MAS as a presenting manifestation in our cohort of childhood lupus

Methods: We retrospectively studied 140 pediatric lupus patients from January 1993- November 2018 and collected clinical and laboratory data of patients (3) who had MAS as presenting manifestation.

Results: Case 1 was 11-year-old girl with fever for 4 months associated with rash and generalized body swelling for 1 month. Examination revealed rash over malar area and ear lobules, anasarca, hepatomegaly, bilateral pleural and pericardial effusion. In view of multisystem involvement a possibility of lupus was considered which was confirmed by investigations (table 1). She had elevated ferritin and fasting triglyceride and low fibrinogen. A clinical possibility of lupus with associated MAS was considered. She received pulses of methylprednisolone, one dose of intravenous immunoglobulin following which she improved. In view of nephrotic range proteinuria she was started on induction regimen with cyclophosphamide and shifted to mycophenolate in maintenance. Her initial SLEDAI-2k was 32- this decreased to 4 at 3 year follow-up.

Case 2 was a 9-year-old girl with fever, rash, generalized body swelling for 1 month and altered sensorium for 4 days. Examination revealed pallor, oral ulcers and hepatomegaly. She was in shock at presentation. In view of multisystem involvement a possibility of lupus was considered which was confirmed by investigations (table 1). She had pericardial effusion and low ejection fraction (25\%). A possibility of MAS was considered and investigations revealed hyperferritinemia, elevated triglyceride and hypofibrinoginemia. She was given methylprednisolone pulses and continued on oral prednisolone, mycophenolate and hydroxychloroquine. Her initial SLEDAI-2k was 17- this decreased to 0 at 3 year follow-up. Case 3 was an 8-year-old girl who had fever, rash and body swelling for 15 days. On examination she had tachycardia, tachypnea, pallor, anasarca, subconjunctival bleed and frontal alopecia. She had pleural and pericardial effusion. In view of multisystem involvement a possibility of lupus was considered which was confirmed by investigations (table1). She had high ferritin and triglyceride. So a possibility of MAS was considered and she received pulse methyl prednisolone and intravenous immunoglobulin. She also had hematuria and proteinuria (renal biopsy could not be performed as she was sick and had thrombocytopenia) so was given pulse cyclophosphamide followed by mycophenolate. Her initial SLEDAI-2k was 25- this decreased to 0 at 2 year follow-up.

Conclusion: MAS can be the presenting manifestation of pediatric lupus and may contribute to disease severity and requires aggressive management.

\section{REFERENCES}

[1] Borgia RE, Gerstein M, Levy DM, Silverman ED, Hiraki LT. Features, Treatment, and Outcomes of Macrophage Activation Syndrome in 